\title{
Corrigendum: Structure and evolution of the serum paraoxonase family of detoxifying and anti-atherosclerotic enzymes
}

Michal Harel, Amir Aharoni, Leonid Gaidukov, Boris Brumshtein, Olga Khersonsky, Ran Meged, Hay Dvir, Raimond B G Ravelli, Andrew McCarthy, Lilly Toker, Israel Silman, Joel L Sussman \& Dan S Tawfik

Nat. Struct. Mol. Biol. 11, 412-419 (2004).

Some of the enzymatic activity data for paraoxonase (PON1) reported in this article were biased by two factors. First, the gene encoding the H115Q mutant contained an additional H177R mutation introduced during PCR amplification. Although His177 is far from the active site and the H177R mutant by itself is 70\% active compared to the wild-type PON1, the combined H115Q H177R mutant is almost completely inactive. Second, the H115Q and the H134Q mutants are unstable and prone to misfolding and thus aggregation.

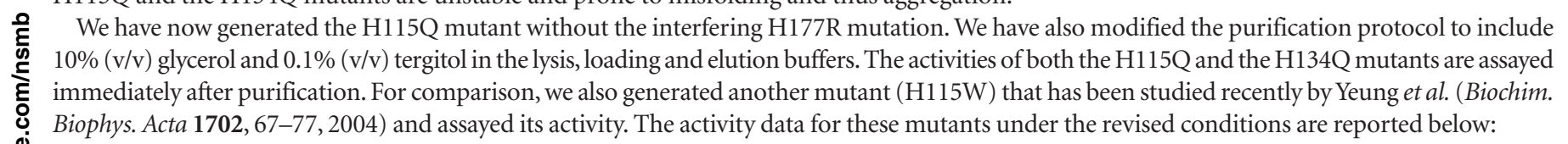

\begin{tabular}{llc} 
Mutation & Aryl-esterase (\% relative to WT) & Paraoxonase (\% relative to WT) \\
\hline H115Q & 0.6 & 32 \\
H115W & 0.056 & 195 \\
H134Q & 9.9 & 600
\end{tabular}

When these same mutations are incorporated into the recombinant PON1 variant G2E6, the structure of which was determined and reported in the article, and into another recombinant PON1 variant (G3C9; see Table 1 of the article), we obtained similar activity data as above (not shown). These results indicate that His 115 and His 134 are responsible for the aryl-esterase activity but not the paraoxonase activity of PON1. Thus, the two hydrolytic activities may be catalyzed by different active site residues, and the model postulated in the article may be valid only for the aryl-esterase activity. We apologize for any inconvenience this may have caused.

\section{Erratum: Observation of internal cleavage and ligation reactions of} a ribozyme

Michelle Nahas, Timothy J Wilson, Sungchul Hohng, Kaera Jarvie, David M J Lilley \& Taekjip Ha

Nat. Struct. Mol. Biol. 11, 1107-1113 (2004).

A mistake was introduced during production in the acknowledgments section (page 1112, column 2, line 45) of the manuscript. The correct acknowledgments should read: "Funding was provided by the US National Science Foundation (NSF) (PHY-0134916, DBI-0215869, T.H.), by the US National Institutes of Health (NIH) (GM065367, T.H.), and by Cancer Research-UK (D.M.J.L.). M.K.N. was partially supported by the NIH molecular biophysics training grant (T32GM008276)." We apologize for any inconvenience this may have caused. 